\title{
Exact Solutions of Two Nonlinear Partial Differential Equations by the First Integral Method
}

\author{
Qingmei Zhang, Mei Xiong, Longwei Chen \\ College of Statistics and Mathematics, Yunnan University of Finance and Economics, Kunming, China \\ Email:1477273038@qq.com,tc715@sina.com,clwxmff@163.com
}

How to cite this paper: Zhang, Q.M., Xiong, M. and Chen, L.W. (2020) Exact Solutions of Two Nonlinear Partial Differential Equations by the First Integral Method. Advances in Pure Mathematics, 10, 12-20.

https://doi.org/10.4236/apm.2020.101002

Received: November 10, 2019

Accepted: January 13, 2020

Published: January 16, 2020

Copyright $\odot 2020$ by author(s) and Scientific Research Publishing Inc. This work is licensed under the Creative Commons Attribution International License (CC BY 4.0).

http://creativecommons.org/licenses/by/4.0/

\begin{abstract}
In recent years, many methods have been used to find the exact solutions of nonlinear partial differential equations. One of them is called the first integral method, which is based on the ring theory of commutative algebra. In this paper, exact travelling wave solutions of the Non-Boussinesq wavepacket model and the $(2+1)$-dimensional Zoomeron equation are studied by using the first integral method. From the solving process and results, the first integral method has the characteristics of simplicity, directness and effectiveness about solving the exact travelling wave solutions of nonlinear partial differential equations. In other words, tedious calculations can be avoided by Maple software; the solutions of more accurate and richer travelling wave solutions are obtained. Therefore, this method is an effective method for solving exact solutions of nonlinear partial differential equations.
\end{abstract}

\section{Keywords}

The First Integral Method, The Partial Differential Equations, The Exact Travelling Wave Solutions

\section{Introduction}

Over the past few decades, finding the exact solutions of nonlinear partial differential equations (PDEs) has become an attractive topic in physical science and nonlinear science. The nonlinear PDE is an important model for describing the problems of fluid mechanics, chemical physics, kinematics, atmosphere and ocean phenomena and so on. In order to find the exact solutions of nonlinear PDEs, pioneers presented the following these methods, such as $\left(G^{\prime} / G\right)$-expansion method [1], tanh-sech function method [2], Darboux and Backlund transform 
[3], bifurcation theory method of dynamic systems [4], Jacobi elliptic function expansion method [5], F expansion method [6], exp-function method [7], Hirota bilinear method [8] and so on.

Since Feng [9] proposed solving nonlinear PDEs by the first integral method. The theory has been applied to handle various PDEs by many scholars in science and engineering. such as S. Ibrahim et al. [10] used the first integral method to obtain the exact solutions of three nonlinear schrodinger equations. S. S. Singh [11] employed the first integral method to solve the exact solutions of Kudryashov-Sinelshchikov equation and generalized Radhakrishnan-Kundu-Lakshmanan equation. A. Seadawy et al. [12] obtain the solitary wave solutions of Cubic-Quintic Nonlinear Schrödinger and Variant Boussinesq Equations by the first integral method. N. Taghizadeh et al. [13] used the first integral method to study the exact solutions of three nonlinear PDEs, etc.

For the Non-Boussinesq wavepacket model, H. Wang et al. [14] obtain the solitary wave solutions, kink and anti-kink wave solutions, periodic travelling wave solutions of the Non-Boussinesq wavepacket model by dynamical systems and bifurcation theories, and the travelling wave solutions are expressed by the hyperbolic functions and Jacobian elliptic functions. P. Robin et al. [15] introduced in this Boussinesq-type model a forcing term and the present numerical results are compared with experiments conducted in the large air/sea facility of IRPHE at Marseille. Furthermore, for the $(2+1)$-dimensional Zoomeron equation, Q.S. Liu and X.Q. Zhou [16] investigated the $(2+1)$-dimensional Zoomeron equation by using the generalized algebraic method, and the Jacobi elliptic function solutions, the hyperbolic function solutions and the trigonometric function solutions are obtained. K. Khan et al. [17] applied an enhanced $\left(G^{\prime} / G\right)$-expansion method to find the traveling wave solutions of the $(2+1)$-dimensional Zoomeron equation. As a result, the hyperbolic and trigonometric functions involving several parameters are derived, and so on.

The rest of this paper is structured as follows. In Section 2, we introduce the basic idea of the first integral method briefly. In Section 3, we use this method to solve two partial differential equations in detail. Finally, a conclusion is given in Section 4 .

\section{The First Integral Method}

Consider the general nonlinear PDE in the form

$$
P\left(u, u_{t}, u_{x}, u_{x x}, u_{t t}, u_{x t}, u_{x x x}, \cdots\right)=0 .
$$

where $P$ is a polynomial in its arguments.

We use the transformations

$$
u(x, t)=u(\xi), \xi=x-c t .
$$

where $\mathrm{c}$ is a constant, then

$$
\frac{\partial}{\partial t}(\cdot)=-c \frac{\partial}{\partial \xi}(\cdot), \frac{\partial}{\partial x}(\cdot)=\frac{\partial}{\partial \xi}(\cdot), \frac{\partial^{2}}{\partial t^{2}}(\cdot)=c^{2} \frac{\partial^{2}}{\partial \xi^{2}}(\cdot), \cdots .
$$


Step 1: We use the Equation (3) to transform the nonlinear PDE (1) into nonlinear ordinary differential equations (ODEs)

$$
Q\left(u, u_{\xi}, u_{\xi \xi}, \cdots\right)=0 .
$$

where the subscript denotes the derivation with respect to $\xi$.

Step 2: Suppose the solution of nonlinear ODE (4) can be translated as

$$
u(x, t)=u(\xi)
$$

Step 3: We introduce a new independent variable

$$
X(\xi)=u(\xi), Y(\xi)=u_{\xi}(\xi) .
$$

which leads to the system of ODEs

$$
\left\{\begin{array}{l}
X_{\xi}(\xi)=Y(\xi) \\
Y_{\xi}(\xi)=F(X(\xi), Y(\xi))
\end{array}\right.
$$

Step 4: According the qualitative theory of ODEs [18], if we can find the first integrals to Equation (7) under the same conditions, then the general solutions to Equation (7) can be found directly. However, there is no systematic theory that can tell us how to find its first integrals, nor we know what these first integrals are. Fortunately, for some equations we can apply the Division Theorem to reduces Equation (4) to a first order integrable ordinary differential equation. An exact solution to Equation (1) is obtained by solving this equation. Now, let us review the Division Theorem [19].

Division Theorem: Suppose that $P(x, y)$ and $Q(x, y)$ are polynomials in $C(x, y)$, and make $P(x, y)$ is irreducible in $C(x, y)$. If $Q(x, y)$ vanishes at all zero points of $P(x, y)$, then there exists a polynomial $F(x, y)$ in $C(x, y)$, such that

$$
Q(x, y)=P(x, y) F(x, y) \text {. }
$$

\section{Application}

In this section, we apply the first integral method based on the Division Theorem to solve the two nonlinear PDEs.

Firstly, we consider the following nonlinear Boussinesq wavepacket model

$$
\begin{aligned}
& i\left(\partial_{t}+c_{g} \partial_{z}\right) A+\frac{\omega^{\prime \prime}}{2} A_{z z}-\frac{N^{2} k^{2} e \frac{z}{H}}{2 \omega} A|A|^{2} \\
& -i\left[\frac{\omega^{\prime \prime \prime}}{6} A_{\mathrm{zzz}}+\frac{\omega}{4}\left(m+\frac{i}{H}\right) A\left(e^{\frac{z}{H}}|A|^{2}\right)_{z}\right]=0,
\end{aligned}
$$

where $N>0, \quad z>0, H>0, H^{-1}=\frac{N^{2}}{g}$, and

$$
\omega^{2}=\frac{N^{2} k^{2}}{k^{2}+m^{2}+H^{-2} / 4}, \quad c_{g}=\omega^{\prime}=\frac{\partial \omega}{\partial m}=-\frac{\omega m}{k^{2}+m^{2}+H^{-2} / 4},
$$




$$
\begin{aligned}
& \omega^{\prime \prime}=\frac{\partial^{2} \omega}{\partial m^{2}}=\frac{\omega\left(2 m^{2}-k^{2}-H^{-2} / 4\right)}{\left(k^{2}+m^{2}+H^{-2} / 4\right)^{2}}, \\
& \omega^{\prime \prime \prime}=\frac{\partial^{3} \omega}{\partial m^{3}}=-\frac{3 \omega m\left(2 m^{2}-3 k^{2}-3 H^{-2} / 4\right)}{\left(k^{2}+m^{2}+H^{-2} / 4\right)^{3}} .
\end{aligned}
$$

Here $k>0, m>0$, let $\omega>0$, we also can get $c_{p}=\frac{\omega m}{k^{2}+m^{2}}>0$ and $c_{g}<0$. The Boussinesq limit in Equation (9) is to let $H \rightarrow \infty$ that is let $\frac{N^{2}}{g} \rightarrow 0$ and retain $N$.

Now let's introduce the following scalings

$$
t=\left(L /\left|c_{g}\right|\right) t_{1}, \quad z=L x_{1}, \quad(k, m)=\left(k_{1}, m_{1}\right) / L, \quad A=2 \sqrt{\left|c_{g}\right| /(\omega m)} u_{1},
$$

where $L=\frac{\omega^{\prime \prime}}{2\left|c_{g}\right|}$.

Substituting Equation (12) into Equation (9) and dropping the mark 1, we get

$$
i\left(\partial_{t}-\partial_{x}\right) u+u_{x x}-2 \sigma \beta e^{\epsilon \sigma x} u|u|^{2}+i\left[\alpha u_{x x x}-(1+i \epsilon \sigma / m) u\left(e^{\epsilon \sigma x}|u|^{2}\right)_{x}\right]=0,
$$

where

$$
\begin{gathered}
\sigma=\operatorname{sgn}\left(\omega^{\prime \prime}\right)=\operatorname{sgn}(\alpha)= \pm 1, \quad \epsilon=|L| / H>0, \\
\alpha=\frac{2 m^{2}\left(2 m^{2}-3 k^{2}-3 \epsilon^{2} / 4\right)}{\left(2 m^{2}-k^{2}-\epsilon^{2} / 4\right)^{2}}, \quad \beta=\frac{\left|2 m^{2}-k^{2}-\epsilon^{2} / 4\right|}{2 m^{2}} \geq 0 .
\end{gathered}
$$

Then the Boussinesq limit in Equation (13) corresponds to $\epsilon=0$ and Equation (13) can be written to

$$
i\left(\partial_{t}-\partial_{x}\right) u+u_{x x}-2 \sigma \beta u|u|^{2}+i\left[\alpha u_{x x x}-u\left(|u|^{2}\right)_{x}\right]=0,
$$

or

$$
i u_{t}-i u_{x}+u_{x x}-2 \sigma \beta u|u|^{2}+i \alpha u_{x x x}-i u\left(|u|^{2}\right)_{x}=0,
$$

Suppose the from of solution to Equation (17) is as following:

$$
u(x, t)=\varphi(\xi) e^{i(p x-q t)} .
$$

where $\xi=\beta x-\lambda t$. And $\beta, \lambda, p, q$ are real parameters, $\varphi(\xi)$ is real functions.

Substituting Equation (18) into Equation (17), and making imaginary and real part are zero respectively:

$$
\begin{gathered}
\alpha \beta^{3} \varphi^{\prime \prime \prime}+\left(2 p \beta-\lambda-\beta-3 p^{2} \beta \alpha\right) \varphi^{\prime}-2 \varphi^{2} \varphi^{\prime}=0, \\
\left(\beta^{2}-3 \alpha p \beta^{2}\right) \varphi^{\prime \prime}+\left(q+\alpha p^{3}-p-p^{2}\right) \varphi-2 \sigma \beta \varphi^{3}=0 .
\end{gathered}
$$

Integrating Equation (19) once, and substituting the resultant equation into Equation (20), we have 
$\varphi^{\prime \prime}+\frac{q+\alpha p^{3}-p-p^{2}+2 p \beta-\lambda-\beta-3 p^{2} \beta \alpha}{\beta^{2}-3 \alpha p \beta^{2}+\alpha \beta^{3}} \varphi-\frac{2 \sigma \beta+\frac{2}{3}}{\beta^{2}-3 \alpha p \beta^{2}+\alpha \beta^{3}} \varphi^{3}=0$.

For simplicity, we denote $\delta_{1}=-\frac{q+\alpha p^{3}-p-p^{2}+2 p \beta-\lambda-\beta-3 p^{2} \beta \alpha}{\beta^{2}-3 \alpha p \beta^{2}+\alpha \beta^{3}}$ and $\delta_{2}=\frac{2 \sigma \beta+\frac{2}{3}}{\beta^{2}-3 \alpha p \beta^{2}+\alpha \beta^{3}}$. Putting Equation (6) in Equation (21), we get

$$
\left\{\begin{array}{l}
X^{\prime}(\xi)=Y(\xi), \\
Y^{\prime}(\xi)=\delta_{1} X(\xi)+\delta_{2} X(\xi)^{3} .
\end{array}\right.
$$

Secondly, we consider the following $(2+1)$-dimensional Zoomeron equation

$$
\left(\frac{u_{x y}}{u}\right)_{t t}-\left(\frac{u_{x y}}{u}\right)_{x x}+\left(2 u^{2}\right)_{x t}=0 .
$$

Suppose the form of solution to Equation (23) is as following:

$$
u(x, y, t)=\phi(\xi), \quad \xi=k x+l y-\lambda t .
$$

where $k, l, \lambda$ are real parameters, $\phi(\xi)$ is real functions.

Substituting Equation (24) into Equation (23), and integrating the resultant equation twice for Equation (23), we obtain

$$
\left(\lambda^{2} k l-k^{2} l^{2}\right) \phi^{\prime \prime}-2 k \lambda \phi^{3}+p \phi=0,
$$

where $p$ is the integration constant.

Taking Equation (6) in Equation (25), we get

$$
\left\{\begin{array}{l}
X^{\prime}(\xi)=Y(\xi) \\
Y^{\prime}(\xi)=-\frac{p}{\lambda^{2} k l-k^{2} l^{2}} X(\xi)+\frac{2 k \lambda}{\lambda^{2} k l-k^{2} l^{2}} X(\xi)^{3}
\end{array}\right.
$$

If we let again $\delta_{1}=-\frac{p}{\lambda^{2} k l-k^{2} l^{2}}, \quad \delta_{2}=\frac{2 k \lambda}{\lambda^{2} k l-k^{2} l^{2}}$ then Equations (22) and (26) is equivalent to the first integral. In order to obtain their exact solution, we just need to talk about system Equation (22).

Now, according to the first integral method. We assume the $X=X(\xi)$ and $Y=Y(\xi)$ are the nontrivial solutions of Equation (22), and

$$
Q(X, Y)=\sum_{i=0}^{n} a_{i}(X) Y^{i}
$$

is an irreducible polynomial in the complex domain $C[X, Y]$ such that

$$
Q(X(\xi), Y(\xi))=\sum_{i=0}^{n} a_{i}(X(\xi)) Y(\xi)^{i}=0 .
$$

where $a_{i}(X)(i=0,1, \cdots, n)$ are polynomial of $X$ and $a_{n}(X) \neq 0$. Equation (28) is called the first integral of the Equation (22). Note that $Q[X(\xi), Y(\xi)]$ is a polynomial in $X$ and $Y$, and $\frac{\mathrm{d} Q}{\mathrm{~d} \xi}$ implies $\left.\frac{\mathrm{d} Q}{\mathrm{~d} \xi}\right|_{(27)}=0$. According the Division 
Theorem, there exists a polynomial $H(X, Y)=h(X)+g(X) Y$ in $C(X, Y)$, such that

$$
\left.\frac{\mathrm{d} Q}{\mathrm{~d} \xi}\right|_{(19)}=\left.\left(\frac{\mathrm{d} Q}{\mathrm{~d} X} \frac{\mathrm{d} X}{\mathrm{~d} \xi}+\frac{\mathrm{d} Q}{\mathrm{~d} Y} \frac{\mathrm{d} Y}{\mathrm{~d} \xi}\right)\right|_{(27)}=(h(X)+g(X) Y)\left(\sum_{i=0}^{N} a_{i}(X) Y^{i}\right) .
$$

In this example, we make two different cases, suppose that $n=1$ and $n=2$ in Equation (28).

\section{Case I}

Assume that $n=1$, and then from Equation (29) we have

$$
\sum_{i=0}^{1} a_{i}^{\prime}(X) Y^{i+1}+\sum_{i=0}^{1} i a_{i}(X) Y^{i-1}\left(Y^{\prime}(\xi)\right)=(h(X)+g(X) Y)\left(\sum_{i=0}^{1} a_{i}(X) Y^{i}\right) .
$$

where prime denotes differentiating with respect to the variable $X$. By comparing with the coefficient of $Y^{i}(i=2,1,0)$ on both sides of Equation (30), we get

$$
\begin{gathered}
a_{1}^{\prime}(X)=g(X) a_{1}(X), \\
a_{0}^{\prime}(X)=h(X) a_{1}(X)+g(X) a_{0}(X), \\
a_{1}(X)\left(\delta_{1} X+\delta_{2} X^{3}\right)=h(X) a_{0}(X) .
\end{gathered}
$$

Since $a_{i}(X)(i=0,1)$ are polynomials, then from Equation (31) we obtain that $a_{1}(X)$ is constant and $g(X)=0$. For simplicity, make $a_{1}(X)=1$. Balancing the degrees of $h(X)$ and $a_{0}(X)$, we get that $\operatorname{deg}(h(X))=1$ only. Assume that $h(X)=A_{0} X+A_{1}$, where $A_{0} \neq 0$, then

$$
a_{0}(X)=\frac{1}{2} A_{0} X^{2}+A_{1} X+f .
$$

where $f$ is an arbitrary integration constant.

Substituting $a_{0}(X), a_{1}(X)$ and $h(X)$ into Equation (33) and setting all the coefficient of powers of $X$ to be zero, then we obtain a system of nonlinear algebraic equations, that is,

$$
\left\{\begin{array}{l}
\frac{1}{2} A_{0}^{2}=\delta_{2} \\
\frac{3}{2} A_{0} A_{1}=0 \\
A_{0} f+A_{1}^{2}=\delta_{1} \\
A_{1} f=0,
\end{array}\right.
$$

and using Maple solving them, we have

$$
A_{1}=0, A_{0}= \pm \sqrt{2 \delta_{2}}, f= \pm \frac{\delta_{1}}{\sqrt{2 \delta_{2}}},
$$

Taking the conditions (36) in Equation (28), we get

$$
Y(\xi)=\mp\left(\frac{1}{2} \sqrt{2 \delta_{2}} X^{2}+\frac{\delta_{1}}{\sqrt{2 \delta_{2}}}\right),
$$

and combining Equation (37) with Equation (22), we can obtain the exact solutions of Non-Boussinesq wavepacket model (9) as 


$$
u(\xi)=\mp \frac{\sqrt{\delta_{1} \delta_{2}}}{\delta_{2}} \tanh \left(\sqrt{\frac{\delta_{1}}{2}} \xi+\xi_{0}\right) .
$$

where $\xi_{0}$ is an arbitrary constant. And the travelling wave solutions of Non-Boussinesq wavepacket model (9) can be written as

$$
u(x, t)=\mp \frac{\sqrt{\delta_{1} \delta_{2}}}{\delta_{2}} e^{(p x-q t)} \tanh \left[\sqrt{\frac{\delta_{1}}{2}}(\beta x-\lambda t)+\xi_{0}\right] .
$$

\section{Case II}

Suppose that $n=2$, by comparing with the coefficient of $Y^{i}(i=3,2,1,0)$ on both sides of Equation (28), we obtain

$$
\begin{gathered}
a_{2}^{\prime}(X)=g(X) a_{2}(X), \\
a_{1}^{\prime}(X)=h(X) a_{2}(X)+g(X) a_{1}(X), \\
a_{0}^{\prime}(X)+2 a_{2}(X)\left(\delta_{1} X+\delta_{2} X^{3}\right)=h(X) a_{1}(X)+g(X) a_{0}(X), \\
a_{1}(X)\left(\delta_{1} X+\delta_{1} X^{3}\right)=h(X) a_{0}(X) .
\end{gathered}
$$

Since $a_{i}(X)(i=2,1,0)=0$ are polynomials, then from Equation (40) we obtain $a_{2}(X)$ is a constant and $g(X)=0$. For simplicity, make $a_{2}(X)=1$. Balancing the degrees of $h(X), a_{1}(X)$ and $a_{0}(X)$, we get $\operatorname{deg}(h(X))=1$, $\operatorname{deg}\left(a_{1}(X)\right)=2, \operatorname{deg}\left(a_{0}(X)\right)=4$ only. Assume $h(X)=A_{0} X+A_{1}$, where $A_{0} \neq 0$, then

$$
\begin{aligned}
a_{1}(X)= & \frac{1}{2} A_{0} X^{2}+A_{1} X+f, \\
a_{0}(X)= & \left(\frac{1}{8} A_{0}^{2}-\frac{1}{2} \delta_{2}\right) X^{4}+\frac{1}{2} A_{0} A_{1} X^{3} \\
& +\left(\frac{1}{2} A_{0} f+\frac{1}{2} A_{1}^{2}-\delta_{1}\right) X^{2}+A_{1} f X+g .
\end{aligned}
$$

where $g$ is an arbitrary integration constant. Substituting $a_{0}(X), a_{1}(X)$, $a_{2}(X)$ and $h(X)$ into Equation (43), setting all the coefficient of powers of $X$ equal to zero, then we obtain a system of nonlinear algebraic equation, that is,

$$
\left\{\begin{array}{l}
\frac{1}{8} A_{0}^{3}=A_{0} \delta_{2} \\
\frac{5}{12} A_{0}^{2} A_{1}=A_{1} \delta_{2} \\
A_{0}^{2} f+2 A_{0} A_{1}^{2}=3 A_{0} \delta_{1}+2 f \delta_{2} \\
A_{0} A_{1} f+A_{1}^{3}=4 A_{1} \delta_{1} \\
A_{1}^{2} f+A_{0} g=f \delta_{1} \\
A_{1} g=0
\end{array}\right.
$$

and using Maple solving them, we get

$$
g=0, f=0, A_{1}=O, A_{0}= \pm 2 \sqrt{2} \sqrt{\delta_{2}},
$$

Taking the conditions (46) in Equation (28), we get 


$$
Y(\xi)=\mp \frac{\sqrt{2}}{2} \sqrt{\delta_{2}} X^{2},
$$

and combining Equation (47) with Equation (22), we can obtain the exact solutions of Non-Boussinesq wavepacket model (9) as

$$
u(\xi)= \pm \frac{\sqrt{2}}{\sqrt{\delta_{2}} \xi+\xi_{0}} .
$$

where $\xi_{0}$ is an arbitrary constant. And the travelling wave solutions of NonBoussinesq wavepacket model (9) can be written as

$$
u(x, t)= \pm \frac{\sqrt{2}}{\sqrt{\delta_{2}}(\beta x-\lambda t)+\xi_{0}} e^{(p x-q t)} .
$$

\section{Conclusion}

In conclusion, we have studied the exact travelling wave solutions of the non-Boussinesq wavepacket model and the $(2+1)$-dimensional Zoomeron equation by the first integral method. By introducing the travelling wave transformations, two nonlinear PDEs have been transformed into ODEs. Then according to the Division Theorem of polynomial, exact travelling wave solutions of two nonlinear PDEs are obtained. Compared with other methods, it is an effective method to solve the exact traveling wave solutions; that is, it avoids complex calculation process by using mathematical software. Therefore, this method can be extended to solve the exact traveling wave solutions of other nonlinear PDEs.

\section{Conflicts of Interest}

The authors declare no conflicts of interest regarding the publication of this paper.

\section{References}

[1] Wang, M., Li, X. and Zhang, J. (2008) The (G'/G)-Expansion Method and Travelling Wave Solutions of Nonlinear Evolution Equations in Mathematical Physics. Physics Letters A, 372, 417-423. https://doi.org/10.1016/j.physleta.2007.07.051

[2] Ma, W.X. (1993) Travelling Wave Solutions to a Seventh Order Generalized KdV Equation. Physics Letters A, 180, 221-224. https://doi.org/10.1016/0375-9601(93)90699-Z

[3] Matveev, V.B. and Salle, M.A. (1991) Darboux Transformation and Solitons. Journal of Neurochemistry, 42, 1667-1676. https://doi.org/10.1007/978-3-662-00922-2

[4] Li, Y., Shan, W.R., Shuai, T.P. and Rao, K. (2015) Bifurcation Analysis and Solutions of a Higher Order Nonlinear Schrödinger Equation. Mathematical Problems in Engineering, 22, 121-125. https://doi.org/10.1155/2015/408586

[5] Liu, S., Fu, Z., Liu, S.D. and Zhao, Q. (2001) Jacobi Elliptic Function Expansion Method and Periodic Wave Solutions of Nonlinear Wave Equations. Physics Letters $A$, 289, 69-74. https://doi.org/10.1016/S0375-9601(01)00580-1

[6] Zhang, S. (2006) The Periodic Wave Solutions for the $(2+1)$ Dimensional Konopelchenko Dubrovsky Equations. Chaos, Solitons \& Fractals, 30, 1213-1220. 
https://doi.org/10.1016/j.chaos.2005.08.201

[7] He, J.H. and Zhang, L.N. (2008) Generalized Solitary Solution and Compacton-Like Solution of the Jaulent-Miodek Equations Using the Exp-Function Method. Physics Letters A, 372, 1044-1047. https://doi.org/10.1016/j.physleta.2007.08.059

[8] Wang, D.S. (2009) A Systematic Method to Construct Hirotas Transformations of Continuous Soliton Equations and Its Applications. Computers and Mathematics with Applications, 58, 146-153. https://doi.org/10.1016/j.camwa.2009.03.077

[9] Feng, Z.S. (2002) The First Integral Method to Study the Burgers Korteweg-de Vries Equation. Journal of Physics A, 25, 343-349. https://doi.org/10.1088/0305-4470/35/2/312

[10] Ibrahim, S. and El-Ganaini, A. (2007) The First Integral Method to the Nonlinear Schrödinger Equations in Higher Dimensions. Abstract and Applied Analysis, 18, 1187-1197.

[11] Singh, S.S. (2006) Solutions of Kudryashov-Sinelshchikov Equation and Generalized Radhakrishnan-Kundu-Lakshmanan Equation by the First Integral Method. International Journal of Physical Research, 4, 37-42. https://doi.org/10.14419/ijpr.v4i2.6202

[12] Seadawy, A. and Sayed, A. (2017) Soliton Solutions of Cubic-Quintic Nonlinear Schrödinger and Variant Boussinesq Equations by the First Integral Method. Faculty of Sciences and Mathematics, 214, 4199-4208. https://doi.org/10.2298/FIL1713199S

[13] Taghizadeh, N., Mirzazadeh, M. and Samiei Paghaleh, A. (2012) The First Integral Method to Nonlinear Partial Differential Equations. Applications and Applied Mathematics, Iran, 102, 117-132.

[14] Wang, H., Chen, L.W. and Liu, H.J. (2015) Nonlinear Dynamics and Exact Travelling Wave Solutions in the Non-Boussinesq Wavepacket Model. International Conference on Fuzzy Systems and Knowledge Discovery, Zhangjiajie, 15-17 August 2015, 2587-2591.

[15] Robin, P., Kimmoun, O. and Kharif, C. (2011) Influence of Wind on Focusing Waves Packet Using a Boussinesq-Type Model.

[16] Liu, Q.S. and Zhou, X.Q. (2014) New Exact Solutions of $(2+1)$-Dimensional Zoomeron Equation. Natural Science Edition of Hebei Normal University, 3.

[17] Khana, K., Akbar, M.A., Salam, M.A. and Islam, M.H. (2014) A Note on Enhanced (G'/G)-Expansion Method in Nonlinear Physics. Ain Shams Engineering, 5, 877-884. https://doi.org/10.1016/j.asej.2013.12.013

[18] Ding, T.R. and Li, C.Z. (1996) Ordinary Differential Equations. Peking University Press, Beijing.

[19] Feng, Z.S. (2002) The First Integral Method to Study the Burgers-Korteweg-de Vries Equation. Journal of Physics A: Mathematical and General, 35, 343-349. https://doi.org/10.1088/0305-4470/35/2/312 